\title{
Conducta clínica frente a la fractura de corono-esmalte y subluxación en dientes primarios: reporte de caso
}

\author{
Conduta clínica frente à fratura corono-esmalte e subluxação em dentes decíduos: relato de caso \\ Clinical management of corono-enamel fracture and subluxation in deciduous teeth: case report
}

\author{
Marcelle DANELON ${ }^{\mathbf{1}, 2}$ \\ Nayara Gonçalves EMERENCIANO ${ }^{1}$ \\ Francyenne Maira Castro GONÇALVES \\ Liliana Carolina Baez QUINTERO' ${ }^{1}$ \\ Alberto Carlos Botazzo DELBEM ${ }^{1}$
}

Departamento de Odontologia Infantil e Social, Faculdade de Odontologia, UNESP Univ. Estadual Paulista, 16015-050 Araçatuba-SP, Brasil Faculdade de Odontologia, UNAERP Universidade de Ribeirão Preto, 14096-900 Ribeirão Preto-SP, Brasil

\section{Resumen}

Introducción: Los traumatismos en los dientes decimales son comunes de ocurrir, siendo su primer episodio normalmente en los niños cuando están aprendiendo a caminar. Los traumas nuevos o repetidos pueden ocurrir a lo largo del crecimiento y desarrollo del niño, siendo importante su diagnóstico, seguimiento y tratamiento cuando sea necesario. Objetivo: El objetivo del presente estudio fue relatar un caso clínico de fractura corono-esmalte y subluxación en dentición decidua, bien como sus complicaciones clínicas, radiográficas y conducta clínica. Relato de caso: Paciente de sexo femenino, 2 años y 8 meses de edad, compareció a la clínica de Odontopediatría de la Facultad de Odontología de Araçatuba (FOA/UNESP), Brasil, cuya mamá relataba la aparición de una "bolita" en la región superior del diente 51 y fractura corono-esmalte. Durante la anamnesis la mamá relato que la niña se había caído hace un mes y golpeado el diente 51 presentando sangramiento, leve movilidad y fractura corono-esmalte, y que según el odontopediatría que atendió la niña en el momento del trauma le informo que se trataba de un traumatismo tipo subluxación, y que la conducta clínica, debería ser acompañamiento. Al examen clínico se observó alteración en la coloración del diente 51 y presencia de fistula en la región. Al examen radiográfico fue posible observar inicio de reabsorción radicular y lesión periapical denotando necrosis pulpar. El plan de tratamiento instaurado fue endodoncia del diente 51, seguido por acompañamiento clínico y radiográfico del mismo. Después de 8 días, la niña retorno sin la presencia de fistula, mostrando la eficacia del tratamiento instaurado. Conclusión: Se concluye, por tanto que aunque la subluxación pueda traer daños al diente deciduo, cuando es diagnosticada precozmente, la alteración es susceptible de tratamiento efectivo y satisfactorio, llevando a la preservación del diente hasta el momento de su exfoliación.

Descriptores: Traumatismos de los Dientes; Diente Primario; Terapéutica.

\section{Resumo}

Introdução: Os traumatismos em dentes decíduos são comuns de ocorrer, sendo seu primeiro episódio normalmente nas crianças quando estão aprendendo a andar. Traumas novos ou repetidos podem ocorrer ao longo do crescimento e desenvolvimento da criança, sendo importante seu diagnóstico, acompanhamento e tratamento quando necessário. Objetivo: O objetivo do presente estudo foi relatar um caso clínico de fratura corono-esmalte e subluxação na dentição decídua, bem como suas complicações clínicas, radiográficas e conduta clínica. Relato de caso: Paciente do sexo feminino, 2 anos e 8 meses de idade, compareceu à clínica de Odontopediatria da Faculdade de Odontologia de Araçatuba (FOA/UNESP), Brasil, cuja mãe relatava o aparecimento de "bolinha" na região superior do dente 51 e fratura corono-esmalte. Durante a anamenese mãe relatou que a criança havia caído há 1 mês e batido o dente 51 apresentando sangramento, leve mobilidade e fratura corono-esmalte, e que segundo a odontopediatra que atendeu a criança no momento do trauma a informou que tratava-se de um traumatismo do tipo subluxação, e que como conduta clínica, deveria ser o acompanhamento. Ao exame clínico observou-se alteração na coloração do dente 51 e presença de fístula na região. Ao exame radiográfico foi possível observar início de reabsorção radicular e lesão periapical denotando necrose pulpar. O plano de tratamento instituído foi o tratamento endodôntico do dente 51, seguido pelo acompanhamento clínico e radiográfico do mesmo. Após 8 dias, a criança retornou sem a presença da fístula, mostrando a eficácia do tratamento insituído. Conclusão: Conclui-se, portanto que embora a subluxação possa trazer danos ao dente decíduo, quando diagnosticada precocemente, a alteração, é passível de tratamento efetivo e satisfatório, levando à preservação do dente até o momento de sua exfoliação.

Descritores: Traumatismos Dentários; Dente Decíduo; Terapêutica.

\section{Abstract}

Introduction: Trauma to primary teeth is common, and is the first episode usually seen in children when they are learning to walk. New or repeated traumas may occur throughout the child's growth and development, and their diagnosis, follow-up and treatment are important when necessary. Aim: The aim of the present study was to report a clinical case of corono-enamel fracture and subluxation in the deciduous dentition, as well as its clinical, radiographic and clinical complications. Case report: A 2-year, 8-month-old female patient attended the Pediatric Dentistry Clinic of the Faculty of Dentistry of Araçatuba (FOA/UNESP), Brazil, whose mother reported the appearance of a "ball" on the upper tooth 51 and a corono-enamel fracture. During the anamnesis, the mother reported that the child had fallen for 1 month and beat the 51 tooth presenting bleeding, mild mobility and corono-enamel fracture, and according to the pediatric dentist who attended the child at the time of the trauma informed her that it was a traumatic type of subluxation, and that as a clinical course, it should be the follow-up. Clinical examination revealed a change in tooth color 51 and presence of fistula in the region. Radiographic examination revealed the beginning of root resorption and periapical lesion denoting pulp necrosis. The treatment plan was the endodontic treatment of tooth 51, followed by clinical and radiographic follow-up. After 8 days, the child returned without the presence of the fistula, showing the efficacy of the treatment. Conclusion: It is concluded, therefore, that although the subluxation can bring damage to the deciduous tooth, when it is diagnosed early, the alteration is capable of effective and satisfactory treatment, leading to the preservation of the tooth until the moment of its exfoliation.

Descriptors: Tooth Injuries; Tooth, Deciduous; Therapeutics.

\section{INTRODUCCIÓN}

En la Odontología, el área responsable por los cuidados de los niños y adolescentes como un todo, de forma a obtenerse un sistema aestomatognatico funcional, equilibrado y estético, es denominada Odontopediatria. Sin embargo, la caries, las enfermedades periodontales y las lesiones traumáticas, amenazan este equilibrio, lo que puede causar desarmonía Costa et al. ${ }^{1}$

El traumatismo dentario (TD) puede ser definido como una agresión térmica, química o 
mecánica sufrida por el diente y estructuras adjacentes, cuya magnitud supera la resistencia encontrada en los tejidos óseos y dentarios. La extensión de las lesiones traumáticas posee una relación directa con la intensidad, tipo y duración del impacto $^{2}$, constituyendo una de las urgencias que más afecta a los pacientes en Odontopediatría, siendo considerada una situación frecuente en los consultorios médicos/odontológicos, y cerca del $40 \%$ de los casos es en niños, siendo en muchos casos el primer motivo de consulta con el odontólogo ${ }^{3,4}$. Son frecuentes cuando el niño comienza a caminar, esto es por vuelta del primer al tercer año de vida, fase en que el niño todavía no posee completa coordinación motora que le permita movimientos seguros y precisos, llevando a caídas, que son las causas más frecuentes asociadas al trauma ${ }^{5}$. Estudios han demostrado picos de incidencia de traumatismo en otros grupos de edad, lo que evidencia que los niños están sujetos a traumatismos en cualquier edad, principalmente antes de los 6 años de vida ${ }^{4,6}$. De acuerdo con Nogueira et $\mathrm{al}^{7}$. las caídas ocupan un lugar en destaque en la etiología de los traumatismos dentarios en la dentición decidua, que vienen de situaciones como andar, correr, pelear, accidentes automovilísticos, juegos, atletismo, choques contra objetos, entre otros ${ }^{8}$. La literatura destaca otros factores predisponentes para el traumatismo dentario como pacientes portadores de clase II división 1 de Angle y Clase I con sobre saliente de maxila ${ }^{9}$, overjet acentuado, incompleto vedamiento labial, protrusión de los incisivos centrales superiores, mordida abierta y obesidad infantil ${ }^{10}$. Además de los factores encima mencionados, la violencia (maltrato) también son relatados en la literatura ${ }^{4,7}$.

La prevalencia de los dientes damnificados son los anteriores superiores, los cuales son los más afectados en la dentición decidua, especialmente el diente $61^{11}$. Los dos géneros son igualmente afectados, siendo observada una tendencia de prevalencia ligeramente mayor en el sexo masculino ${ }^{4,12}$. Sin embargo, Fried e Erickson ${ }^{13}$ relatan en una revisión de literatura que la incidencia de los traumatismos en la dentición decidua vario de 4 a $30 \%$, siendo dependiente de la evaluación, y del tipo de estudio donde los dientes más traumatizados serían los anteriores y los superiores, con un mayor compromiso de ambos incisivos centrales superiores, mostrando todavía que ambos géneros presentan un elevado índice de trauma principalmente entre las edades de 4 a 5 años.

La clasificación de los traumatismos dentarios es bastante importante y sirve de guía para auxiliar en la elaboración de un diagnóstico, tanto en la dentición decidua como en la dentición permanente, proporcionando no sólo una orientación para el tratamiento sino también un posible pronostico. Podemos dividir os TD basados en la clasificación de Andreasen et al. ${ }^{4}$, aceptada por la Organización Mundial de la Salud ${ }^{3,14}$ en: a) Lesiones de tejido duro dentario (líneas de fractura en esmalte, fractura de esmalte, fractura de esmalte y dentina, fractura de esmalte y dentina con exposición pulpar, fractura radicular) y b) Lesión de los tejidos de soporte de los dientes (contusión, subluxación, luxación lateral, luxación intrusiva, luxación extrusiva y avulsión).

Dentro de las consecuencias del TD en la dentición decidua se puede destacar secuelas tanto en los dientes traumatizados, así como en sus sucesores. Los dientes deciduos que sufrieron traumatismo pueden presentar hemorragia pulpar, alteración del color, perdida prematura, obliteración de la cámara pulpar, necrosis pulpar $^{3,15}$ y reabsorción interna y externa. Ese tipo de reabsorción se puede presentar en la superficie externa o interna en el diente siendo contrario a la reabsorción fisiológica. Radiográficamente se observa discontinuidad de la lámina dura, además de la ocurrencia de un padrón acelerado cuando se comparan ambas ${ }^{4,8,15,16}$. Dentro de las lesiones antes destacadas, las fracturas de esmalte y subluxación ocurren con cierta frecuencia en la dentición decidua ${ }^{8}$. En estos casos la literatura preconiza como tratamiento el recubrimiento del esmalte o apenas alivio oclusal. Ya para la subluxación se recomienda orientación dietética y restricción del uso de chupetes y teteros; en caso haya movilidad acentuada una contención flexible o semirrígida deberá ser instalada por un tiempo de 10 a 14 días, para la comodidad del paciente ${ }^{8,17}$.

De esta forma, para evitar mayores daños a los dientes deciduos traumatizados y sucesores permanentes es necesario que se tomen cuidados y atenciones propias al trauma. El diagnostico debe ser rápido y preciso basándose en los hallazgos clínicos y radiográficos. Aunque las radiografías son consideradas como exámenes complementarios, son esenciales para auxiliar al profesional en el diagnóstico, así como en el control posterior al trauma, objetivando detectar precozmente alteraciones de anormalidad, tanto en el diente afectado como en su sucesor permanente ${ }^{3,18}$.

Ese trabajo tiene como proposición relatar un caso clínico de subluxación con fractura coronoesmalte en la dentición decidua, bien como sus complicaciones clínicas, radiográficas y conducta clínica.

\section{REPORTE DE CASO}

Paciente de género femenino, 2 años y 8 meses de edad, compareció al consultorio de Odontopediatría particular, cuya madre relataba el aparecimiento de "bolita" en la región superior del diente $51 \mathrm{y}$ fractura corono-esmalte. Al examen clínico se observó alteración en la coloración del diente 51 y presencia de fistula en la región 
(Figura 1). Al examen radiográfico fue posible observar el inicio de una reabsorción radicular y lesión periapical denotando necrosis pulpar (Figura 2).

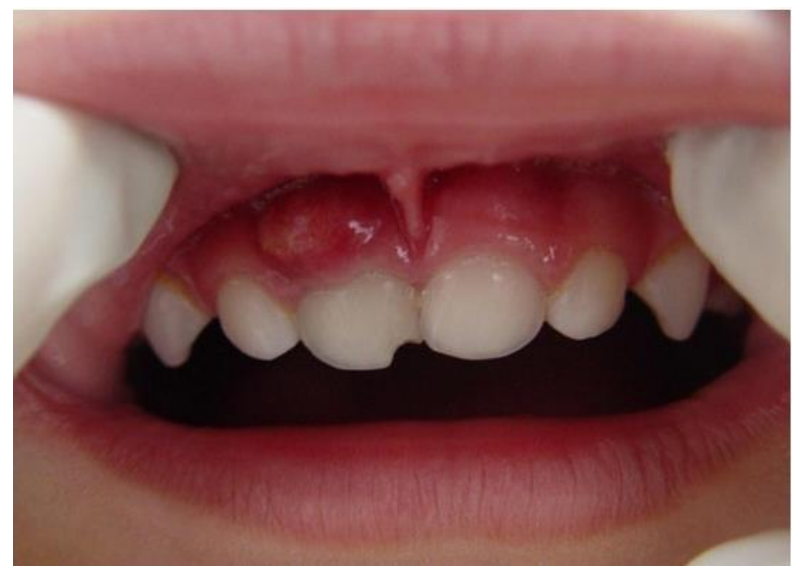

Figura 1: Aspecto clínico inicial.

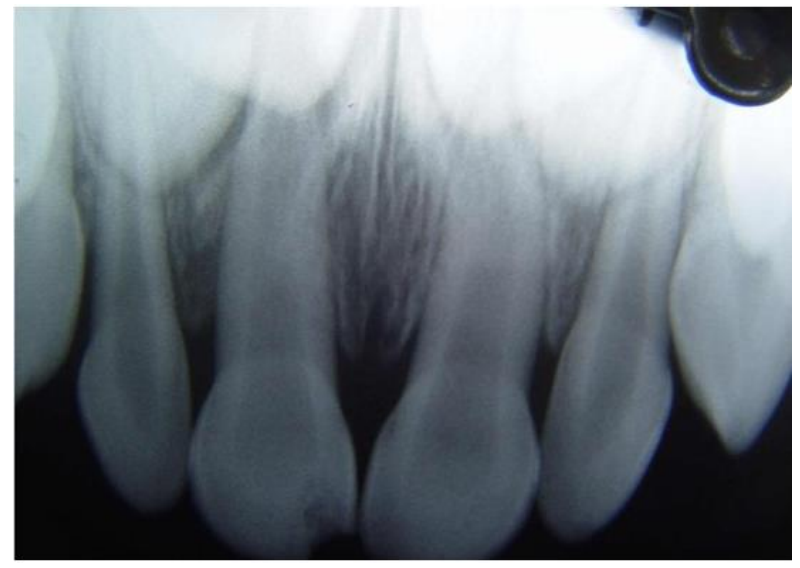

Figura 2: Aspecto clínico inicial.

El plan de tratamiento instituido fue el tratamiento de endodoncia del diente 51, seguido por el acompañamiento clínico y radiográfico del mismo. Después de los cuidados pre-operatorios, se realizó la anestesia tópica del área con Benzotop (NOVA DFL $($ ), por 3 minutos seguida por la anestesia terminal infiltrativa en la región vestibular y palatina con Citocaina al $3 \%$.

Después del aislamiento absoluto del diente 51 se procede con la abertura coronaria utilizando una fresa de carburo esférica \# 4, como se muestra en la Figura 3. A continuación, con el auxilio de una lima Kerr \#15 se procedió con la remoción de la pulpa radicular (Figura 4).

Durante todo el momento de la remoción de la pulpa radicular, se procedió también con la irrigación del canal radicular con hipoclorito de sodio al 2,5\%, debido a la presencia de la lesión periapical (Figura 5). Después, se procedió con el secado del canal radicular utilizando puntas de papel absorbente (Tanari®). En la secuencia se procede con la instrumentación y preparo biomecánico del canal radicular del diente 51, con lima Kerr \# 15, \# 20, \# 25 y \# 30, y constante irrigación con hipoclorito de sodio al 2,5\% (Figura 6).

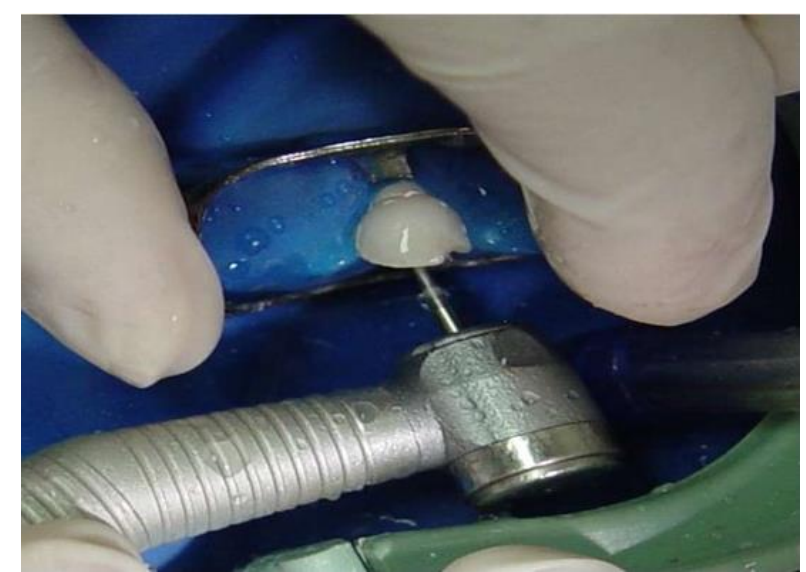

Figura 3: Abertura coronaria utilizando unas fresa de carburo esférica\#4.

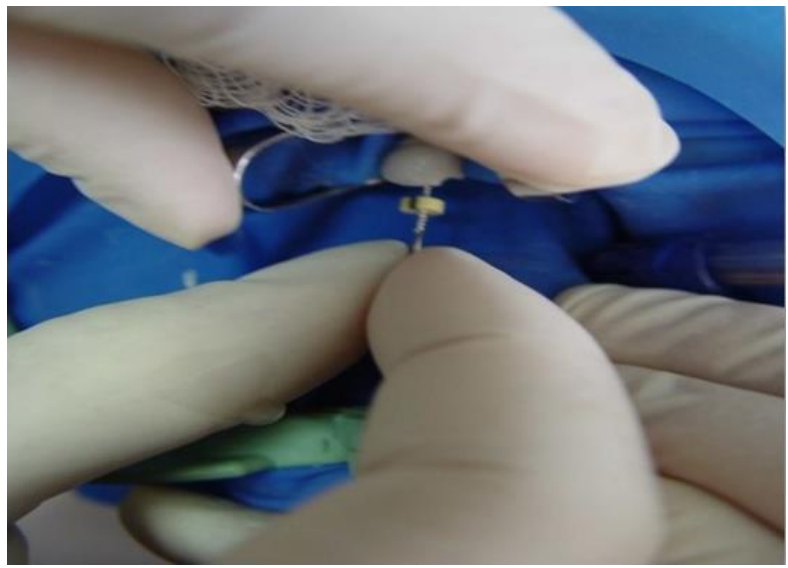

Figura 4: Remoción de la pulpa radicular diente 51 con lima Kerr\#15.

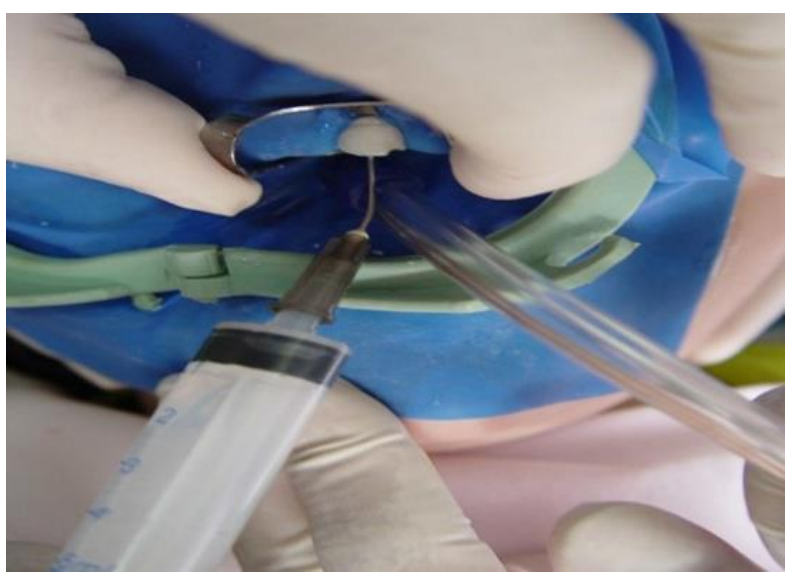

Figura 5: Irrigación del canal radicular con hipoclorito de sódio al 2.5\%.

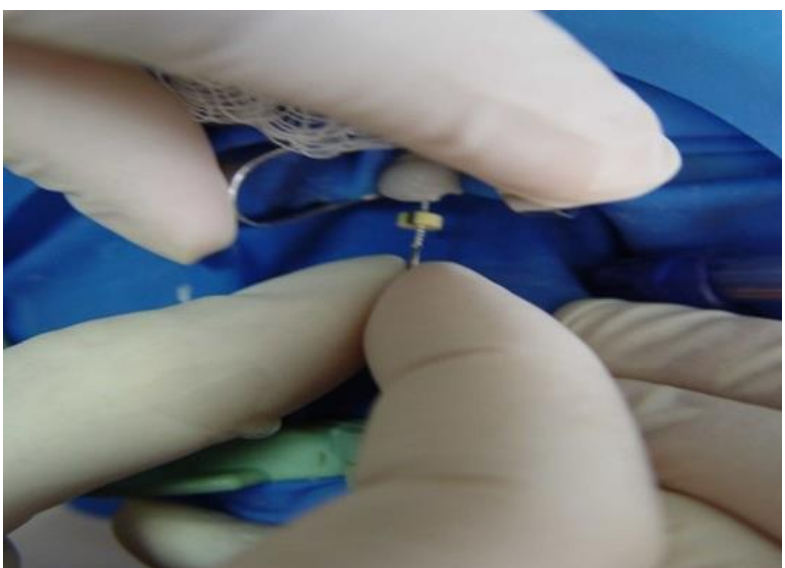

Figura 6: Instrumentación y preparo biomecánico del canal radicular del diente 51 com lima Kerr\#15, \#20, \#25 y \#30. 
Se procedió con el secado del canal radicular utilizando puntas de papel absorbente (Tanariß) y se colocó Pasta de Hidróxido de Calcio Calen PMCC (SS White ${ }^{\circledR}$ ) como curativo de demora, el cual fue cambiado 2 veces con intervalos de 15 dias cada cambio, en el diente 51 (Figura 7). Después de los curativos de demora con Pasta de Hidróxido da Calcio Calen PMCC (SS White ${ }^{\circledR}$ ), la obturación del canal radicular del diente 51 fue realizada utilizando como material obturador Pasta de Hidróxido de Calcio Calen espesada con óxido de zinc (OZ) (SS White $^{\circledR}$ ) (Figura 8), seguido por la restauración de la cara palatina con resina compuesta Opallis $\left(\mathrm{FGM}^{\circledR}\right)$ color A1 (Figura 9).

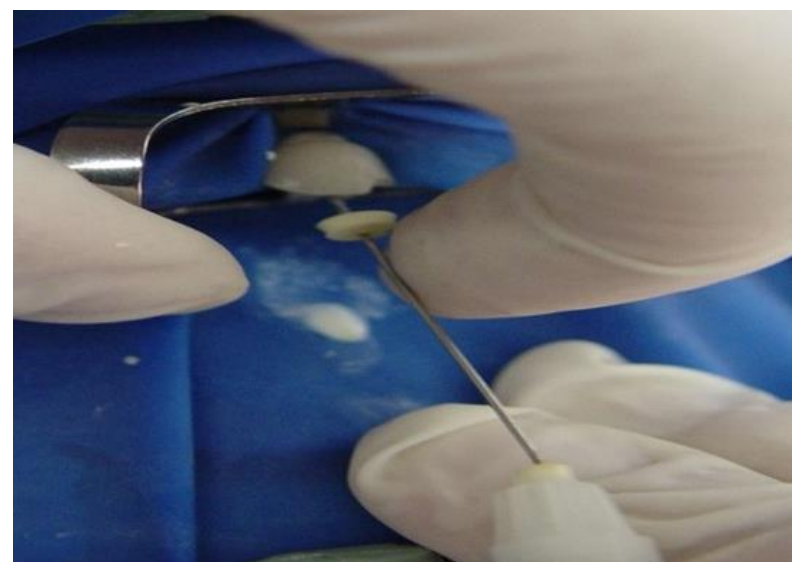

Figura 7: Inserción de pasta de hidróxido de calcio Calen PMCC (SSWhite $\left.{ }^{\circledR}\right)$ como curativo de demora (2x/15dias) diente 51.

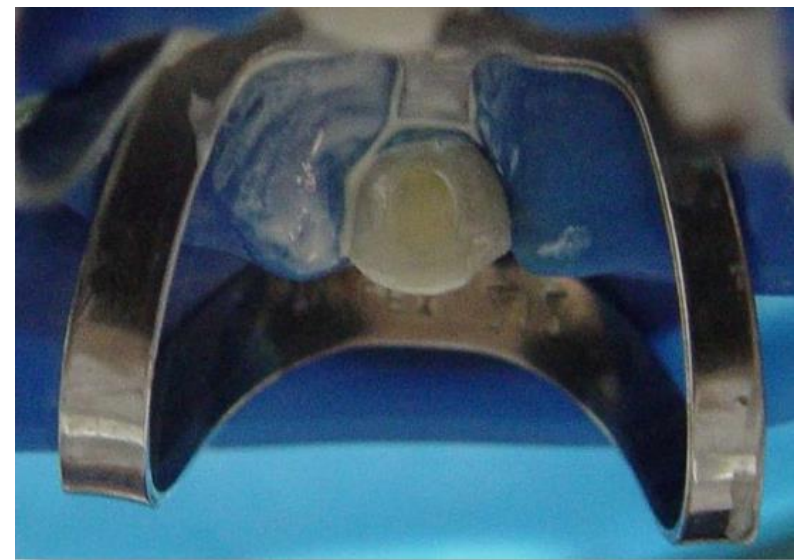

Figura 8: Obturación del canal radicular diente 51 con pasta de hidróxido de calcio Calen espesada con OZ (SSWhite®).

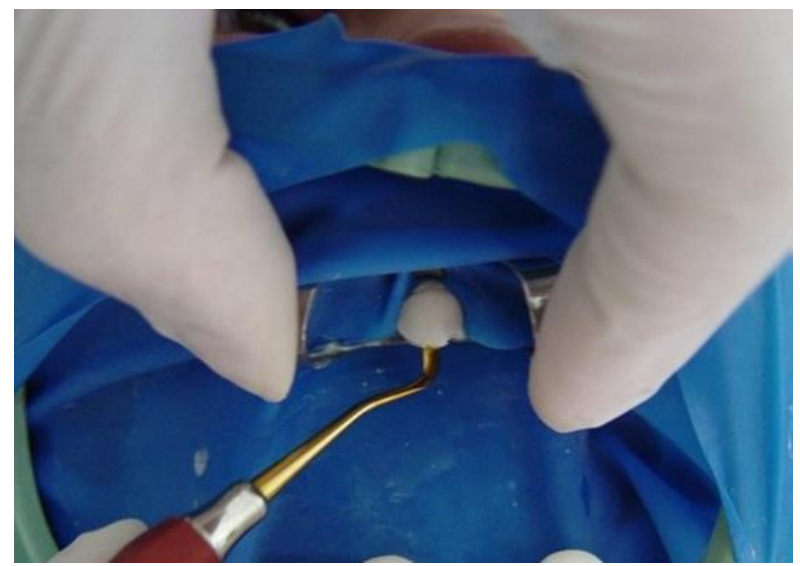

Figura 9: Restauración cara palatina del diente 51 con resina compuesta Opallis (FGM®) color A1.
La figura 10 muestra el aspecto clínico final del diente 51, después de 8 días de la finalización del tratamiento de endodoncia, evidenciando ausencia de fistula en la región del diente 51 y el aspecto radiográfico final del respectivo diente (Figuras 10 y 11).

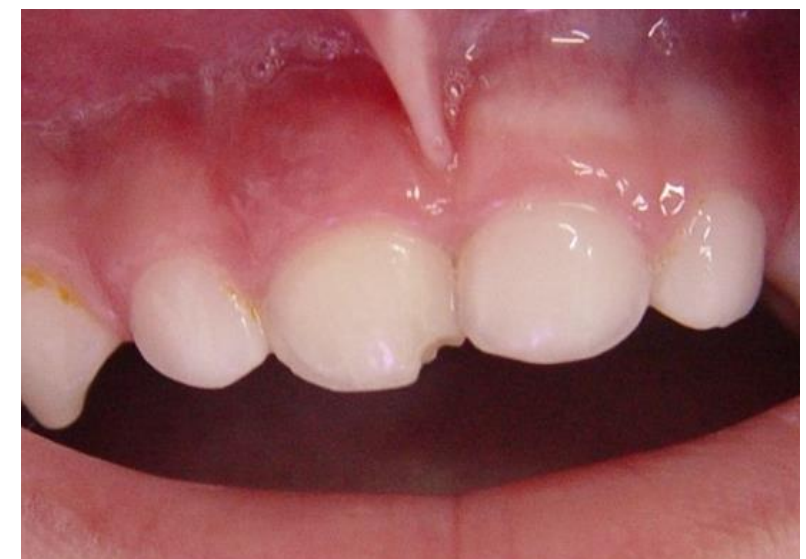

Figura 10: Aspecto clínico final después de 8 días de la finalización de tratamiento de endodoncia.

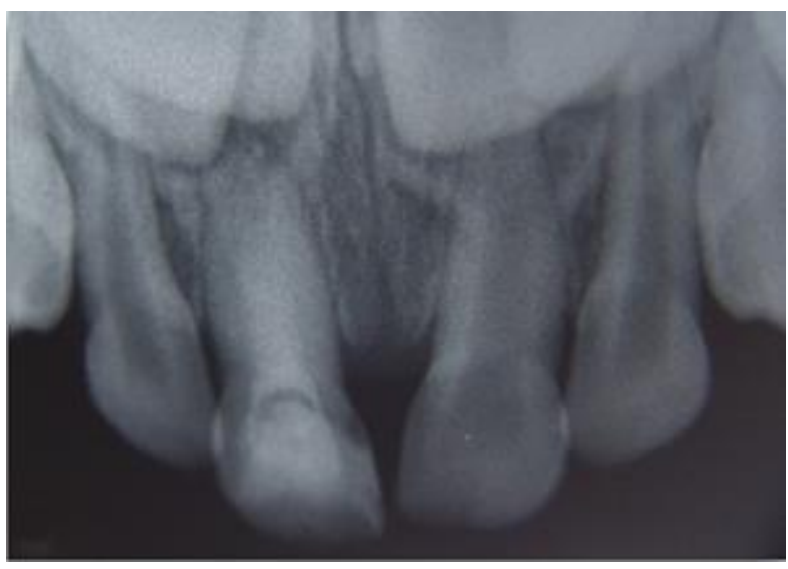

Figura 11: Aspecto radiográfico final

\section{DISCUSSION}

Este trabajo presenta un caso clínico de injuria traumática muy común en niños en la dentición decidua. También describe el diagnóstico y plan de tratamiento del diente traumatizado, presentando como consecuencia reabsorción radicular, lesión periapical y fistula.

El traumatismo en la dentición decidua es un problema grave considerado una situación de urgencia, una vez que, además de los problemas dentarios y sus consecuencias inmediatas y futuras, envuelve al niño y sus papás emocionalmente, lo que no siempre favorecerá el tratamiento inmediato. Chelotti et al. ${ }^{19}$ evidenciaron que el control de la ansiedad de los papás es un factor básico para el tratamiento de pacientes que sufren traumatismos dentarios. Hay relatos en la literatura que la incidencia más alta de trauma dental es observada en niños en la etapa etaria de 1 a 3 años de edad, una vez que presentan reflejos poco desarrollados, siendo la caída de la propia altura la causa más frecuente, conforme es descrito por Kramer et al. ${ }^{20}$ e Turgut et 
al. ${ }^{21}$ y debido a que la resiliencia del tejido óseo es menor en la fase de dentición decidua. Así, los dientes más afectados son los incisivos centrales superiores, alcanzando una tasa de $80 \%$ de los $\operatorname{casos}^{22}$.

Los dientes traumatizados sufren varias secuelas desencadenando consecuencias para ambas denticiones (decidua y permanente) ${ }^{6,23,24}$. En los dientes deciduos entre las principales consecuencias se puede encontrar hiperemia pulpar, calcificación de los conductos radiculares, retención prolongada, reabsorciones interna y externa, anquilosis dental y decoloración coronal $^{25}$. La decoloración coronal todavía es uno de los tipos de secuelas que más se observa después de traumatismos dentales siendo que la frecuencia varia de 25 a $63 \%{ }^{26}$, que puede estar asociado a necrosis pulpar $^{14}$. Sin embargo, esta no debe ser un factor decisivo en el diagnóstico de la misma en dientes deciduos traumatizados. Cuando el diente traumatizado presenta decoloración, el acompañamiento debe ser en intervalos más cortos entre las consultas especialmente en el primer año después del trauma ${ }^{28}$. Además, el abordaje precoz de intervención por tratamiento de endodoncia, cuando la raíz se encuentra radiográficamente intacta, no garantiza mejores resultados a largo plazo, cuando se compara con un abordaje no intervencionista ${ }^{29}$. Sin embargo, en algunas situaciones puede ocurrir en casos más severos la pérdida temprana del diente deciduo. El diagnóstico de reabsorción radicular externa podrá ser detectado a través de algunos signos radiográficos como ensanchamiento del ligamento periodontal, pérdida ósea y reabsorción radicular patológica, como se observó en el presente caso clínico, llevando a la conducta de tratamiento de endodoncia y en casos más severos exodoncia del diente afectado.

Cardoso e Rocha $^{26}$ diagnosticaron que la reabsorción puede ocurrir en un periodo de 46 días a 4 meses después del trauma, siendo que después de 1 año, se constató una frecuencia de aproximadamente $67 \%$ en dientes con traumatismo de grados clasificados como medios a severos. Ya la reabsorción radicular inflamatoria generalmente ocurre después de la necrosis pulpar, y se no es tratada lleva a la perdida temprana de dientes traumatizados $^{29,30}$. Conforme a la gravedad $y$ severidad del trauma acaban por desencadenar daños en el paquete neurovascular, resultando en la reducción de revascularización del tejido pulpar y subsecuente necrosis pulpar. Consecuentemente el tejido dañado estimulará una reacción inflamatoria y la activación de osteoclastos y reabsorción ósea ${ }^{26,31}$. Así la pérdida de vitalidad pulpar es una complicación subsecuente a traumatismos dentales, especialmente después luxaciones severas, siendo observada una frecuencia menor entre los pacientes por debajo de 3 años de edad ${ }^{14}$. El riesgo de necrosis pulpar en dientes con subluxación fue bajo $(8,3 \%)$ en el estudio de Lauridsen et al. ${ }^{32}$ Sin embargo, Quassem et al. ${ }^{33}$ encontraron una frecuencia mayor o semejante a la frecuencia de necrosis pulpar observada en dientes con intrusión. Los autores sugieren que esos resultados pueden ser parcialmente explicados por el retraso en el atendimiento como es observado en el presente caso clínico. En muchos casos dientes con subluxación no mostraron síntomas después de una semana, así es posible que pacientes con atención odontológica tardía, después de ese periodo presentaron manifestaciones clínicas más graves de subluxación con reabsorción avanzada, como se observa en el estudio de Pugliesi et al. ${ }^{34}$, donde la mitad de los pacientes procuraron tratamiento inicial después de dos semanas del trauma. De esa forma el tipo de trauma también influenciará en el desarrollo de la degeneración pulpar. En el estudio de Keowood e $\mathrm{Seow}^{35}$ fue observada mayor prevalencia de la de vitalidad pulpar en traumatismos del tipo dislocamiento. En el trabajo de Soporowski et al. ${ }^{36}$ se constató un gran porcentaje de dientes con trauma del tipo luxaciones que permanecieron con vitalidad pulpar. De esa forma, el establecimiento de la condición pulpar es una tarea difícil, y solamente un acompañamiento a largo plazo va a auxiliar en la conducta apropiada Diangelis et al. ${ }^{37}$

Los tratamientos propuestos en la literatura para pacientes que sufrieron trauma dental consiste en minimizar posibles consecuencias para los dientes deciduos y de igual forma, reducir disturbios de desarrollo de los gérmenes de los sucesores permanentes, incluyendo monitoreo y acompañamiento radiográfico. De acuerdo con las orientaciones de la Academia Americana de Odontopediatría (AAPD) ${ }^{8}$ exámenes clínicos y radiográficos deben ser realizados en los siguientes plazos: Una semana, 3-4 semanas, 6-8 semanas, 6 meses, 1 año y durante cada subsequente hasta la exfoliación dental. En el caso abordado por este estudio, a partir del análisis de los exámenes clínico y radiográfico, fue indicado como tratamiento inmediato la endodoncia del elemento 51 y acompañamiento de los dos elementos dentales (51 y 61). No hay consenso en la literatura sobre el mejor tratamiento para la dentición decidua traumatizada, además niños que presentan lesiones dentales ni siempre son traídas inmediatamente a la atención odontológica. En cuanto algunos autores defienden la exodoncia, otros destacan la importancia de un abordaje más conservador, aumentando la sobrevida de los dientes deciduos siempre que sea posible ${ }^{38,39}$, como es observado en este caso clínico.

\section{CONCLUSION}

El traumatismo dental en niños ocurre con relativa frecuencia ocasionando serias 
complicaciones de orden morfológico, fisiológico y emocional, debiendo el profesional estar preparado para resolver o minimizar problemas inmediatos y tardíos que vengan a ocurrir. Es importante correlacionar manifestaciones clínicas con alteraciones radiográficas. De la misma forma, el conocimiento sobre cómo identificar la necrosis pulpar puede facilitar el acompañamiento de dientes traumatizados, para un correcto diagnóstico y plan de tratamiento, mejorando así el pronóstico en este caso.

Además de esto la historia del trauma y la etiología demostraron que la prevención de estos eventos está fuera del control de muchas familias, una vez que normalmente ocurren en casa, en situaciones cotidianas. Así, cabe al cirujano dentista el papel de esclarecer la necesidad de acompañamiento profesional en la verificación de posibles alteraciones que pueden presentarse después del trauma dental, muchas veces desconocidos por las familias.

\section{REFERENCIAS}

1. Costa VP, Goettems ML, Baldissera EZ, Bertoldi $\mathrm{AD}$, Torriani DD. Clinical and radiographic sequelae to primary teeth affected by dental trauma: a 9- years restrospective study. Braz oral res. 2016; 30(1). pii: S180683242016000100702 .

2. Duarte DA, Bönecker MS, Sant'Anna GR, Suga SS. Lesões traumáticas em dentes decíduos: Tratamento e controle. São Paulo: Ed. Santos; 2001.

3. Flores MT, Malmgren B, Andersson L, Andreasen JO, Bakland LK, Barnett $\mathrm{F}$ et al. Guidelines for the management of traumatic dental injuries. III. Primary teeth. Dent Traumatol. 2007; 23(4):196202.

4. Andreasen JO, Andreasen FM, Andersson L. Text-book and Colour Atlas of Traumatic Injuries to the Teeth. 4th ed. Copenhagen: Blackwell Munksgaard; 2007.

5. Aldrigui JM, Abanto J, Carvalho TS, Mendes FM, Wanderley MT, Bönecker $\mathrm{M}$ et al. Impact of dental injuries and malocclusions on quality of life of young children. Health Qual Life Outcomes. 2011; 9:78.

6. de Amorim Lde F, Estrela C, da Costa LR. Effects of traumatic dental injuries to primary teeth on permanent teeth: a clinical follow-up study. Dental Traumatol. 2011; 27(2):117-21.

7. Nogueira AJ, Nogueira R, Gillet A. Clinical aspects of dental trauma in early childhood. $\mathrm{j}$ bras odontopediatr odontol Bebê. 1999; 2:112-20.

8. American Academy of Pediatric Dentistry (AAPD). Guidelines for the Management of Traumatic Dental Injuries: 3. Injuries in the Primary Dentition. 2017/2018.
9. Norton E, O'Connell AC. Traumatic dental injuries and their association with malocclusion in the primary dentition of Irish children. Dent Traumatol. 2012; 28(1):81-6.

10.Soriano EP, Caldas AF Jr, Góes PS. Risk factors related to traumatic dental injuries in Brazilian schoolchildren. Dental Traumatol. 2004; 20(5):246-50.

11. Wendt FP, Torriani DD, Assunção MC, Romano AR, Bonow ML, Costa CT et al. Traumatic dental injuries in primary dentition: epidemiological study among preschool children in South Brazil. Dental Traumatol. 2010; 26(2):168-73.

12.Jesus MAD, Antunes LAA, Risso PDA, Freire MV, Maia LC. Epidemiologic survey of traumatic dental injuries in children seen at the Federal University of Rio de Janeiro, Brazil. Braz oral res. 2010; 24(1):89-94.

13.Fried J, Erickson P. Anterior tooth trauma in the primary dentition: Incidence, classification, treatment methods, and sequelae: A review of the literature. ASDC J Dent Child.1995; 62(4):256-61.

14. Malmgren B, Andreasen JO, Flores MT, Robertson A, Diangelis AJ, Andersson L, et al. Guidelines for the management of traumatic dental injuries: 3 . Injuries in the primary dentition. Pediatr Dent. 2017; 39(6):420-28.

15. Borges TS, Chaffee BW, Kramer PF, Feldens EG, Vítolo MR, Feldens CA. Relationship between overweight/obesity in the first year of age and traumatic dental injuries in early childhood: Findings from a birth cohort study. 2017; 33(6):465-71.

16. Soares TRC, Silva LP, Salazar SLA, Luiz RR, Risso PA, Maia LC. Profile of intrusive luxation and healing complications in deciduous and permanent teeth - a retrospective study. Acta Odontol Scand. 2018; 76(8):567-71.

17.Machado JP, Lam XT, Chen JW. Use of a clinical decision support tool for the management of traumatic dental injuries in the primary dentition by novice and expert clinicians. Dent Traumatol. 2018; 34(2):120-28.

18. Skaare AB, Aas AL, Wang NJ. Enamel defects on permanent sucessors following luxation injuries to primary teeth and carers'experiences. Int $\mathbf{J}$ Paediatr Dent. 2015; 25(3):221-228.

19. Chelotti A, Valentin C, Prokopowitsch I, Wanderley MT. Lesões traumáticas em dentes decíduos e permanentes jovens. In: Guedes-Pinto AC. Odontopediatria. 7.ed. São Paulo: Santos, 2003; p.649-687.

20.Kramer PF, Zembruski C, Ferreira SH, Feldens CA. Traumatic dental injuries in Brazilian preschool children. Dent Traumatol. 2003; 19(6):299-303. 
21.Turgut MO, Tekçiçek M, Canoglu H. An unusual developmental disturbance of an unerupted permanent incisor due to trauma to its predecessor - a case report. Dent Traumatol. 2006;22(5):28386.

22.Firmino RT, Siqueira MBLD, Vieira-Andrade RG, Gomes GB, Martins CC, Paiva SM et al. Prediction factors for failure to seek treatment following traumatic dental injuries to primary teeth. Braz oral res. 2014; 28(1):1-7.

23.Campos V, Santo, Jácomo DRDE, De Carvalho VC. Q. TraumatIsmo nos dentes decíduos anterIores: estudo longItudInal retrospectIvo com duração de 8 anos. Arq Bras Odontol. 2010; 4(2):61-6.

24.Güngör HC, Püsman E, Uysal S. Eruption delay and sequelae in permanent incisors following intrusive luxation in primary dentition: a case report. Dent Traumatol. 2011;27(2):156-58.

25. Assunção LRDS, Ferelle A, Iwakura MLH, Nascimento LSD, Cunha RF. Luxation injuries in primary teeth: a retrospective study in children assisted at an emergency service. Braz oral res. 2011;25(2):150-56.

26. Cardoso M, Rocha MJ. Federal University of Santa Catarina follow-up management routine for traumatized primary teeth-part 1 . Dent Traumatol. 2004;20(6):307-17.

27.Cardoso M, Rocha MJ. Identification of factors associated with pathological root resorption in traumatized primary teeth. Dent Traumatol. 2008; 24(3):343-49.

28. Holan G. Long-term effect of different treatment modalities for traumatized primary incisors presenting dark coronal discoloration with no other signs of injury. Dent Traumatol. 2006; 22(1):14-7.

29.Holan G. Development of clinical and radiographic signs associated with dark discolored primary incisors following traumatic injuries: a prospective controlled study. Dent Traumatol. 2004; 20(5):276-87.

30. Cordeiro MMR, Santos BZ, Reyes-Carmona JF, Figuereido CP. Primary teeth show less protecting factors against root resorption. Int J Paediatr Dent. 2011; 21(5):361-68.

31.Barnett F. The role of endodontics in the treatment of luxated permanent teeth. Dent Traumatol. 2002; 18(2):47-56.

32.Lauridsen E, Blanche P, Amaloo C, Andreasen J. $\mathrm{O}$. The risk of healing complications in primary teeth with concussion or subluxation injury-A retrospective cohort study. Dent Traumatol. 2017; 33(5):337-44.

33. Quassem A, Goettems M, Torriani DD, Pappen FG. Radicular maturity level of primary teeth and its association with trauma sequelae. Dent Traumatol. 2014; 30(3):227-31 .
34.Pugliesi DM, Cunha RF, Delbem AC, Sundefeld ML. Influence of the type of dental trauma on the pulp vitality and the time elapsed until treatment: a study in patients aged 0-3 years. Dent Traumatol. 2004; 20(3):139-42.

35. Keowood M, Seow WK. Sequalae of trauma to the primary dentition. J Pedod. 1989; 13(3):230-38.

36. Soporowski NJ, Allred EN, Needleman HL. Luxation injuries of primary anterior teethprognosis and related correlates. Pediatr Dent. 1994; 16(2):96-101.

37.Diangelis AJ, Andreasen JO, Ebeleseder KA, Kenny DJ, Trope M, Sigurdsson A et al. Guidelines for the Management of Traumatic Dental Injuries: 1. Fractures and Luxations of Permanent Teeth. Pediatr Dent. 2017; 39(6):401-411.

38.Flores MT. Traumatic injuries in the primary dentition. Dent Traumatol. 2002;18(6):287-89.

39. Cunha RF, Pugliese DM, Percinoto C. Treatment of traumatized primary teeth: a conservative approach. Dent Traumatol. 2007; 23(6):360-63.

\section{CONFLITO DE INTERESSES}

Os autores declaram não haver conflitos de interesse.

\section{AUTOR PARA CORRESPONDENCIA}

Marcelle Danelon

marcelledanelon@hotmail.com

Submetido em 20/08/2018

Aceito em 04/10/2018 